Fields Institute Communications

Volume 00, 0000

\title{
Environment induced localization and superselection rules in a gas of pyramidal molecules
}

\author{
Giovanni Jona-Lasinio \\ Dipartimento di Fisica and INFN \\ Università di Roma "La Sapienza" \\ Piazzale A. Moro 2, Roma 00185, Italy \\ gianni.jona@roma1.infn.it \\ Carlo Presilla \\ Dipartimento di Fisica and INFM \\ Università di Roma "La Sapienza" \\ Piazzale A. Moro 2, Roma 00185, Italy \\ carlo.presilla@roma1.infn.it \\ Cristina Toninelli \\ Dipartimento di Fisica, Università di Roma "La Sapienza", \\ Piazzale A. Moro 2, Roma 00185, Italy \\ cristina.toninelli@roma1.infn.it
}

\begin{abstract}
We propose a model to describe a gas of pyramidal molecules interacting via dipole-dipole interactions. A cooperative effect induced by the interaction modifies the tunneling properties between the classical equilibrium configurations of the single molecule. The model suggests that, for sufficiently high gas density, the molecules become localized in these classical configurations. On this basis it is possible to explain the shift and the disappearance of the inversion line observed upon increase of the pressure in a gas of ammonia or deuterated ammonia. The same mechanism also accounts for the presence of stable optical activity of certain pyramidal molecules. We discuss the concept of environment induced superselection rule which has been invoked in connection with this problem.
\end{abstract}

\section{Introduction}

In this work we consider the behavior of gases of pyramidal molecules, i.e. molecules of the kind $\mathrm{XY}_{3}$ like for example ammonia $\mathrm{NH}_{3}$, deuterated ammonia $\mathrm{ND}_{3}$, phosphine $\mathrm{PH}_{3}$ and arsine $\mathrm{AsH}_{3}$. We begin by presenting an outline of the physical problem and of its history that dates back to the early developments of quantum mechanics.

2000 Mathematics Subject Classification. 81V55; 82B26.

Work supported in part by Cofinanziamento MURST protocollo MM02263577_001.

(C)0000 American Mathematical Society 
For the single pyramidal molecule, owing to the great differences of characteristic energies and times, we can perform some adiabatic approximations. The electronic motion can be separated from the nuclear one (Born-Oppenheimer approximation). We can further separate the one-dimensional motion of inversion of the nucleus $X$ across the plane containing the three nuclei $Y$. The form of the effective potential for this motion is a double well which is symmetric with respect to the inversion plane 11, 2. The corresponding non degenerate eigenstates must be symmetric or anti-symmetric with respect to the inversion plane. Due to tunneling across the finite potential barrier, these eigenstates are delocalized in the two minima and, for energies below the barrier height, are grouped in doublets, i.e. couples of states with a relative splitting in energy small in comparison with the distance from the rest of the spectrum. For the pyramidal molecules under consideration, the thermal energy $k_{B} T$ at room temperature is much smaller than the distance between the first and the second doublet so that we can reduce the problem to the study of a two-level system corresponding to the symmetric and anti-symmetric states of the first doublet.

The existence of delocalized stationary states is clearly in disagreement with the usual chemical view which, relying upon classical theory, considers the molecules as objects with a well defined spatial structure. In particular, for the molecules under consideration the classical view predicts one of the two pyramidal configurations corresponding to the nucleus $X$ localized in one of the wells of the inversion potential.

The quantum prediction of stationary delocalized states implies the presence of a line in the absorption spectrum, the so called inversion line, at a frequency $\bar{\nu}=h^{-1} \Delta E$, where $\Delta E$ is the energy splitting of the first doublet. The experimental results are strongly dependent on the different kind of atoms that form the pyramidal molecule. Experiments performed with $\mathrm{NH}_{3}$ and $\mathrm{ND}_{3}$ reveal the existence of an inversion line, while this line is not found in the case of $\mathrm{AsH}_{3}$ and $\mathrm{PH}_{3}$. These results cannot be considered a demonstration of the existence of degenerate classical states for arsine and phosphine. The values of the energy splitting for these molecules is so small, see Table 1, that the inversion line, even if it existed, would not be detectable. However, there is a stronger evidence suggesting the existence of stationary localized states, at least for arsine (similar experiments are, as far as we know, not available in the case of phosphine). It has been found that some molecules of the kind $A s Y W Z$, i.e. molecules obtained by substituting hydrogens with different atoms, show stable optical activity [3]. This means that the stationary states of the $A s H W Z$ molecule are chiral, i.e. they cannot be superimposed on their mirror image by a rotation, in agreement with the classical prediction of localized pyramidal states. The discrepancy between the presence of optical activity for some kind of molecules and the quantum prediction of delocalized stationary states was pointed out for the first time in 4 and is usually referred to as Hund paradox or chirality problem.

The frequency $\bar{\nu}$ of the inversion line measured in $\mathrm{NH}_{3}$ and $N D_{3}$ gases shows


$h^{-1} \Delta E$ at $P \simeq 0, \bar{\nu}(P)$ decreases by increasing $P$ and vanishes at a critical pressure $P_{c r} \simeq 2 \mathrm{~atm}$ for $N_{3}$ and $P_{c r} \simeq 0.1 \mathrm{~atm}$ for $N D_{3}$. No quantitative theory has been proposed so far for this phenomenon.

As early as 1949, in a short qualitative paper [8] Anderson made the hypothesis that dipole-dipole interaction may induce a localization of the molecular states. In 


\begin{tabular}{l|c|c|c|c} 
& $\mathrm{NH}_{3}$ & $\mathrm{ND}_{3}$ & $\mathrm{PH}_{3}$ & $\mathrm{AsH}_{3}$ \\
\hline$\Delta E\left(\mathrm{~cm}^{-1}\right)$ & 0.81 & $0.53 \times 10^{-1}$ & $3.34 \times 10^{-14}$ & $2.65 \times 10^{-18}$ \\
\hline$\mu($ Debye $)$ & 1.47 & 1.47 & 0.58 & 0.20 \\
\hline
\end{tabular}

Table 1 Energy splitting of the first doublet $\triangle E$ and electric dipole moment $\mu$ for the molecules $\mathrm{NH}_{3}, \mathrm{ND}_{3}, \mathrm{PH}_{3}, \mathrm{AsH}_{3}$ [2]. We remind that $1 \mathrm{~cm}^{-1}=$ $1.984 \times 10^{-23} \mathrm{~J}$ and 1 Debye $=3.33 \times 10^{-30} \mathrm{Cm}$.

this way the important idea was introduced that inter-molecular interactions may be responsible for the observed phenomena. Thirty years later Pfeifer studied a two-level system modeling a molecule which has an almost degenerate ground state and is coupled to the quantized electromagnetic field [9]. He argued that the coupling of the molecule to the radiation field yields two symmetry-breaking effective ground states belonging to two different sectors of the Hilbert space separated by a superselection rule. These states are localized and one is the mirror image of the other. Later it was argued that this localization cannot take place at finite temperature [10, 11, 12].

A quantitative discussion of the effects induced by coupling a single molecule to the environment constituted by the other molecules of the gas was made in [13, 14]. In this work it was shown that, due to the instability of tunneling under weak perturbations [15, 16], the order of magnitude of the molecular dipole-dipole interaction may account for localized ground states in the case of arsine and phosphine while delocalized states were predicted for ammonia. Therefore, the appearance of chirality was interpreted as a collective effect in a system of infinitely many degrees of freedom. This suggests that a phase transition may be invoked to explain the behavior of different pyramidal molecules or the behavior of the same kind of molecule under variation of thermodynamic parameters like pressure.

We have implemented this idea by constructing a simplified model of a gas of pyramidal molecules which exhibits the desired properties and allows a direct comparison with experimental data. Our model predicts, for sufficiently high intermolecular interactions, the presence of two degenerate ground states corresponding to the different localizations of the molecules. This means that there is a quantum phase transition for a critical value of density (or pressure in the case of constant temperature). A study at non zero temperature shows that this transition affects the behaviour of the gas at room temperature and gives a reasonable explanation of the experimental results [5, 6, 7]. In particular, it provides a correct description of the disappearance of the inversion line of $\mathrm{NH}_{3}$ and $\mathrm{ND}_{3}$ on increasing the pressure.

In the present paper, after a description of the model, we discuss its mean field approximation and the appearance of a degeneracy for the ground state. We then analyze the linear response to an external field at zero temperature to obtain the low energy spectrum and its dependence on the inter-molecular interaction. This shows that the frequency of the inversion line decreases upon increasing density and vanishes at the critical density for which the ground state becomes degenerate. For a treatment at finite temperature and a detailed comparison with experiments we refer to our paper [17].

In the last section we discuss the interpretation of the chirality problem in terms of a superselection rule. 


\section{Description of the model}

As we mentioned in the introduction, for an isolated molecule $X Y_{3}$ we can adiabatically separate the electronic motions from the nuclear ones. The latter essentially reduce to the rotational motion of the molecule, the vibrations of the nuclei around their equilibrium positions and the inversion motion of the nucleus $X$ across the plane of the nuclei $Y$. At room temperature the rotational and vibrational degrees of freedom are much faster then the inversion motion and can be taken into account through a renormalization of the potential barrier for the inversion motion. We can further reduce the inversion dynamics to that of a two-level system corresponding to the ground and first excited eigenstates of the corresponding symmetric double well potential. For a general reference see [1].

Consider now a gas of interacting molecules. If the motion of the centers of mass of the molecules is slow in comparison with the two-level dynamics of each isolated molecule, the translational degrees of freedom can be considered frozen. In this case we can reduce our model to a lattice of unit length $\ell=\varrho^{-1 / 3}$, where $\varrho$ is the gas density, with a two-state system at each site. By evaluating the inversion time $\hbar \Delta E^{-1}$ with the data of Table 1 , we see that the lattice approximation is reasonable in the case of $\mathrm{NH}_{3}$ and $\mathrm{ND}_{3}$ up to room temperature. This approximation has been discussed in general [18] and we refer to this paper also for references to previous related works.

In order to define a suitable Hamiltonian for our lattice model, let us begin with the isolated two-level system. We choose the Hamiltonian operator on the two-state Hilbert space to be

$$
-\frac{\Delta E}{2} \sigma^{x}
$$

where $\sigma^{x}$ is the Pauli matrix

$$
\sigma^{x}=\left(\begin{array}{cc}
0 & 1 \\
1 & 0
\end{array}\right)
$$

The corresponding eigenvectors are

$$
\begin{aligned}
|1\rangle & =\frac{1}{\sqrt{2}}\left(\begin{array}{c}
1 \\
1
\end{array}\right) \\
|2\rangle & =\frac{1}{\sqrt{2}}\left(\begin{array}{c}
1 \\
-1
\end{array}\right),
\end{aligned}
$$

with eigenvalues $-\Delta E / 2$ and $+\Delta E / 2$, respectively. For $N$ independent two-state systems, the Hamiltonian is

$$
H_{0}=\sum_{i=1}^{N}-\frac{\Delta E}{2} 1_{1} \otimes 1_{2} \otimes \ldots \otimes \sigma_{i}^{x} \otimes \ldots 1_{N},
$$

where the subscript indicates the single particle space to which the operators are applied.

In order to modeling the interaction among the molecules we briefly recall the discussion made in [13]. If we consider the whole gas it is possible that, owing to some fluctuation in the state of the system, a single molecule becomes localized. This molecule consequently acquires an electric dipole moment $\boldsymbol{\mu}$ that polarizes the surrounding gas. The latter, in its turn, produces an electric reaction field $\boldsymbol{E}_{R}$, collinear with $\boldsymbol{\mu}$, so that the interaction $-\boldsymbol{\mu} \cdot \boldsymbol{E}_{R}$ tends to stabilize the localized 
state of the initial molecule. In other words, there appears a cooperative effect which tends to stabilize the localization. To mimic this effect in the Hilbert space where $H_{0}$ acts, we choose the interaction Hamiltonian

$$
H_{\text {int }}=\sum_{i=1}^{N} \sum_{j=i+1}^{N} g(i, j) 1_{1} \otimes \ldots \otimes \sigma_{i}^{z} \otimes \ldots \otimes \sigma_{j}^{z} \otimes \ldots \otimes 1_{N},
$$

where $g(i, j)=g(j, i)<0$ and $\sigma^{z}$ is the Pauli matrix

$$
\sigma^{z}=\left(\begin{array}{cc}
1 & 0 \\
0 & -1
\end{array}\right)
$$

whose eigenvectors are the localized states

$$
\begin{aligned}
& |L\rangle=\left(\begin{array}{l}
1 \\
0
\end{array}\right)=\frac{1}{\sqrt{2}}(|1\rangle+|2\rangle) \\
& |R\rangle=\left(\begin{array}{l}
0 \\
1
\end{array}\right)=\frac{1}{\sqrt{2}}(|1\rangle-|2\rangle),
\end{aligned}
$$

with eigenvalues +1 and -1 , respectively. The total Hamiltonian

$$
H=H_{0}+H_{\text {int }}
$$

contains two competing terms with $H_{\text {int }}$ which tends to favor stationary localized states while $H_{0}$ favors delocalization. According to the classical dipole-dipole interaction formula, we expect $g(i, j)$ to be proportional to the square of the electric dipole moment $\mu$ of the molecules and to decrease with the cube of the distance

$$
|g(i, j)| \propto \frac{\mu^{2}}{\ell^{3}|i-j|^{3}} .
$$

In the context of a mean field approximation, it is useful to introduce the parameter

$$
G \equiv-\sum_{j=1}^{N_{c}} g(i, j)
$$

wher $N_{c}$ is a cut-off. By neglecting border effects and using (2.11), we write

$$
G=C \frac{\mu^{2}}{\ell^{3}}
$$

where $C$ is a positive constant which will be considered as a phenomenological parameter to be deduced, in the case of $\mathrm{NH}_{3}$ and $\mathrm{ND}_{3}$, from comparison with the experimental data on the inversion line.

\section{Ground state in the mean field approximation}

The mean field approximation for the ground state corresponds to minimizing the energy functional $\langle\psi|H| \psi\rangle$ within the class of states of the form

$$
|\psi\rangle=|\lambda\rangle \ldots|\lambda\rangle
$$

where

$$
|\lambda\rangle=a|1\rangle+b|2\rangle
$$

and

$$
|a|^{2}+|b|^{2}=1 .
$$


This problem reduces to the solution of the non linear eigenvalue equation

$$
h(\lambda)|\lambda\rangle=\eta(\lambda)|\lambda\rangle
$$

where $h(\lambda)$ is the self-consistent single particle Hamiltonian

$$
h(\lambda)=-\frac{\Delta E}{2} \sigma^{x}-G \sigma^{z}\left\langle\lambda\left|\sigma^{z}\right| \lambda\right\rangle .
$$

The non linear term in (3.5) accounts for the interaction of a single molecule with the reaction field and represents the polarization energy of the gas.

We find $|\lambda\rangle$ by solving Eq. (3.4) and evaluate the energy functional $\langle\psi|H| \psi\rangle$ with the corresponding product states (3.1). For the states which minimize $\langle\psi|$ $H|\psi\rangle$ we obtain the following results. If $G<\Delta E / 2$ there is only one mean-field ground state

$$
\left|\psi_{0}\right\rangle=|1\rangle \ldots|1\rangle
$$

with energy

$$
E_{0}=\left\langle\psi_{0}|H| \psi_{0}\right\rangle=-N \frac{\Delta E}{2} .
$$

If $G \geq \Delta E / 2$ there are two degenerate mean-field ground states

$$
\begin{aligned}
\left|\psi_{0}^{L}\right\rangle & =\left|\lambda^{L}\right\rangle \ldots\left|\lambda^{L}\right\rangle \\
\left|\psi_{0}^{R}\right\rangle & =\left|\lambda^{R}\right\rangle \ldots\left|\lambda^{R}\right\rangle,
\end{aligned}
$$

where

$$
\begin{aligned}
\left|\lambda^{L}\right\rangle & =\sqrt{\frac{1}{2}+\frac{\Delta E}{4 G}}|1\rangle+\sqrt{\frac{1}{2}-\frac{\Delta E}{4 G}}|2\rangle \\
\left|\lambda^{R}\right\rangle & =\sigma^{x}\left|\lambda^{L}\right\rangle
\end{aligned}
$$

with energy

$$
E_{0}=-N \frac{\Delta E}{2}-\frac{N}{2 G}\left(\frac{\Delta E}{2}-G\right)^{2}
$$

By defining the critical value

$$
G_{c r}=\frac{\Delta E}{2},
$$

we distinguish the following two cases. For $G \in\left(0, G_{c r}\right)$ the ground state of the system is approximated by a condensate of delocalized symmetric single particle states corresponding to the ground state of an isolated molecule. For $G \in\left(G_{c r}, \infty\right)$ we have two different condensates which approximate the ground state of the system. The corresponding single particle states transform one into the other under the action of the inversion operator $\sigma^{x}$, as shown by Eq. (3.11), and, for $G \gg G_{c r}$, they become localized

$$
\begin{aligned}
\lim _{\frac{\Delta E}{G} \rightarrow 0}\left|\lambda^{L}\right\rangle & =|L\rangle \\
\lim _{\frac{\Delta E}{G} \rightarrow 0}\left|\lambda^{R}\right\rangle & =|R\rangle .
\end{aligned}
$$


The above results suggest the existence of a quantum phase transition at $G=$ $G_{c r}$. According to Eq. (2.13), this phase transition can be obtained by increasing the density $\varrho$ of the gas above the critical value

$$
\varrho_{c r}=\frac{\Delta E}{2} \frac{1}{\mu^{2} C} .
$$

In the next Section we will discuss the behavior of the inversion line obtained in the range $0<\varrho<\varrho_{c r}$ when the gas, still being in the region characterized by delocalization, approaches the phase transition.

\section{Absorption spectrum}

When the gas is exposed to an electro-magnetic radiation of frequency $\nu_{0}=$ $\omega_{0} / 2 \pi$, we add to the Hamiltonian (2.10) the perturbation

$$
H_{e m}(t)=\epsilon f(t) S
$$

where $\epsilon$ is a small parameter,

$$
f(t)=\theta(t) \cos \left(\omega_{0} t\right)
$$

$\theta(t)$ being the Heaviside function, and $S$ is a time independent operator. In the long-wavelength approximation, $\lambda_{0} \gg d$, where $d$ is the molecule size, we have

$$
S=\sum_{i=1}^{N} 1_{1} \otimes \ldots \otimes \sigma_{i}^{z} \otimes \ldots \otimes 1_{N} .
$$

At zero temperature the absorption spectrum induced by the electro-magnetic perturbation consists in a series of lines of frequency $\nu_{n}=\left(E_{n}-E_{0}\right) h^{-1}$ corresponding to transitions of the system from the ground state of $H$ to the $n$-th excited level. The corresponding transition probability is proportional to the square of the matrix element

$$
M_{0 n} \equiv\left\langle\psi_{0}|S| \psi_{n}\right\rangle
$$

Since we are not able to determine exactly the eigenvalue problem $H \psi_{n}=E_{n} \psi_{n}$ for the $N$ particle Hamiltonian (2.10), we will find an approximate mean-field solution in the framework of the linear response theory.

Let us define the function

$$
\mathcal{S}(t) \equiv\langle\psi(t)|S| \psi(t)\rangle,
$$

where $|\psi(t)\rangle=\exp \left[-i\left(H+H_{e m}\right) t / \hbar\right]\left|\psi_{0}\right\rangle$. By expanding $\mathcal{S}(t)$ in powers of $\epsilon$, for the first order term $\mathcal{S}_{1}(t)$ we find [19]

$$
\tilde{\mathcal{S}}_{1}(\omega)=\tilde{f}(\omega) \mathcal{R}(\omega)
$$

where $\tilde{\mathcal{S}}_{1}(\omega)$ and $\tilde{f}(\omega)$ are the Fourier transforms of $\mathcal{S}_{1}(t)$ and $f(t)$ and

$$
\mathcal{R}(\omega)=\sum_{n}\left|M_{0 n}\right|^{2}\left[\frac{1}{\hbar \omega-\left(E_{n}-E_{0}\right)+i \delta}-\frac{1}{\hbar \omega+\left(E_{n}-E_{0}\right)+i \delta}\right]
$$

with $\delta \rightarrow 0^{+}$. The frequencies of the spectroscopic lines and the associated transition probabilities are therefore given by the poles and the residues of $\mathcal{R}(\omega)$. In order to determine $\mathcal{R}(\omega)$, we evaluate $\tilde{\mathcal{S}}_{1}(\omega)$ in a mean-field approximation and then use (4.6). 
The mean-field approximation for the time-dependent state $|\psi(t)\rangle$ is obtained by extremizing the action functional

$$
\int_{0}^{T}\left\langle\psi(t)\left|-i \hbar \partial_{t}+H+H_{e m}\right| \psi(t)\right\rangle d t
$$

in the class of states of the form

$$
|\psi(t)\rangle=|\lambda(t)\rangle \ldots|\lambda(t)\rangle
$$

The single-particle state $|\lambda(t)\rangle$ is thus determined by the nonlinear Schrödinger equation

$$
i \hbar \frac{d|\lambda(t)\rangle}{d t}=\left[h(\lambda(t))+\epsilon f(t) \sigma^{z}\right]|\lambda(t)\rangle,
$$

where $h(\lambda)$ is given by $(3.5)$. Let us assume that the gas is in the quantum region $G \in\left(0, G_{c r}\right)$, so that the mean-field ground state is $\left|\psi_{0}\right\rangle=|1\rangle \ldots|1\rangle$. Since $|\psi(0)\rangle=$ $\left|\psi_{0}\right\rangle$, Eq. (4.10) has to be solved with the initial condition

$$
|\lambda(0)\rangle=|1\rangle \text {. }
$$

The calculation shows that $\mathcal{R}(\omega)$ has a single pole corresponding to a line in the absorption spectrum with frequency

$$
\bar{\nu}=\frac{\Delta E}{h} \sqrt{1-\frac{2 G}{\Delta E}} .
$$

The residue of $\mathcal{R}(\omega)$ at this pole is

$$
\frac{N}{\sqrt{1-\frac{2 G}{\Delta E}}}
$$

Equation (4.12) shows a dependence of $\bar{\nu}$ on the coupling constant $G$. For $G=0$ we have $\bar{\nu}=\Delta E h^{-1}$, namely the inversion line frequency expected from the quantum theory of an isolated molecule. In the region $0<G<G_{c r}, \bar{\nu}$ decreases by increasing $G$ and vanishes for $G=G_{c r}$. In figure 1 we show the described behavior of the inversion line frequency as a function of the gas density $\varrho=G / C \mu^{2}$.

The residue (4.13) multiplied by the photon energy $h \bar{\nu}$ provides, up to a constant, the intensity $I$ of the inversion line

$$
I \propto h \bar{\nu} \frac{N}{\sqrt{1-\frac{2 G}{\Delta E}}}=N \Delta E .
$$

Note that the divergence at $G=G_{c r}$ shown by the residue is cancelled by the factor $h \bar{\nu}$ which vanishes in the same limit.

\section{Optical activity and superselection rules}

Our model does not distinguish molecules of the type $X Y_{3}$ from the substituted derivatives $X Y W Z$. In fact, we believe that this distinction should not matter for the disappearance of the inversion line. However, an important difference between the two cases is that for $X Y_{3}$ the localized states can be obtained one from the other either by a rotation or by space inversion, while for $X Y W Z$ they can be connected only by space inversion. This implies that $X Y W Z$ molecules, whenever become localized, i.e. at a density greater than the critical value, are chiral and must show stable optical activity. Stable optical activity is commonly observed for 


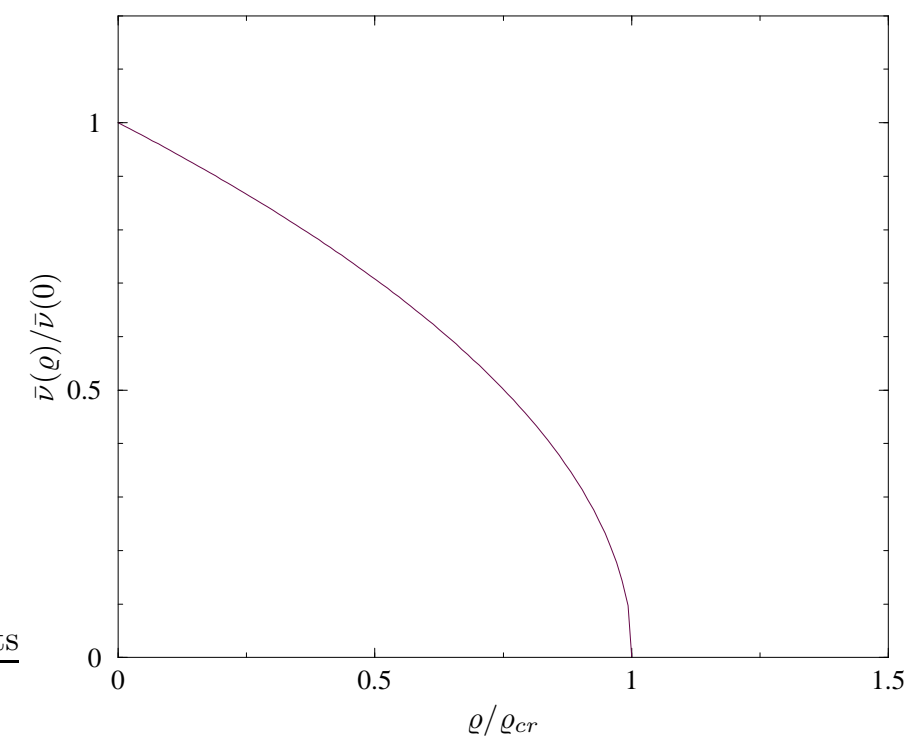

Figure 1 Inversion-line frequency $\bar{\nu}$ as a function of the gas density $\varrho$.

some substituted derivatives of $\mathrm{AsH}_{3}$ for which the critical density is exceedingly small. For the theory of optical activity we refer, for example, to [20].

To explain the stability of the optical activity, i.e. why the localized states are stable, although they are not eigenstates of the single-molecule Hamiltonian, an environment induced superselection rule has been invoked [9, 10, 11, 12.

Following 11, 12 we say that in a system with infinitely many degrees of freedom there is a superselection rule between two different sectors of a Hilbert space if there are neither spontaneous transitions between their state vectors, nor any measurable quantity with matrix element different from zero between them. This implies that any coherent superposition of states among the two sectors is physically meaningless and that we can construct a classical observable (i.e. an observable with zero dispersion on all the states) which acquires the same value on all the vectors of each sector. Examples are the charge and the Boson-Fermion superselection rules. The first refers to the impossibility of coherent superpositions of states with different charge, the second to the impossibility of superposing states with integer and half-integer angular momentum.

With respect to this definition, in the limit of an infinite number of molecules (thermodynamic limit) our model has a superselection rule similar to that found by Pfeifer analyzing a single molecule interacting with the radiation field [9]. Above the critical density, the Hilbert space separates into two sectors generated by the ground state vectors given in mean field approximation by (3.9). These sectors, which we call $\mathcal{H}_{L}$ and $\mathcal{H}_{R}$, cannot be connected by any operator involving a finite number of degrees of freedom (local operator). Let us define chirality $\chi=\lim _{N \rightarrow \infty} \frac{1}{N} \sum_{i=1}^{N} 1_{1} \otimes \ldots \otimes \sigma_{i}^{z} \otimes \ldots \otimes 1_{N}$. In the limit $N \rightarrow \infty$ we have $\chi \psi= \pm \psi$ for $\psi$ in $\mathcal{H}_{L}$ and $\mathcal{H}_{R}$, respectively. Optical activity is expected when $\chi \neq 0$. 
From the above definition the concept of superselection rule is applicable only to pure states. A superselection rule, however, puts some restrictions in the construction of ensembles (density matrices) because these cannot contain mixed terms corresponding to vectors belonging to different sectors of the Hilbert space. Indeed, at finite temperature, we find that in the mean field approximation the free energy of our model is minimized by two density matrices which have vanishing matrix elements between the two sectors $\mathcal{H}_{L}$ and $\mathcal{H}_{R}$ [17. Any other admissible density matrix will be a convex combination of these two.

A different point of view consists in considering, instead of the whole gas, a subsystem composed by a single molecule or a finite number of them still interacting with the rest of the gas. In this case a superselection rule in the Hilbert space of the subsystem can emerge dynamically. By this we mean that there exist projection operators such that the off-diagonal contributions of the subsystem density matrix vanish when time $t \rightarrow \infty$ 21]. This alternative point of view, usually referred to as environment induced superselection rule [22], can be presumably implemented in our model if we describe the interaction of a molecule with the rest of the gas by means of a Lindblad equation [23].

It is a pleasure to dedicate this paper to Sergio Doplicher on the occasion of his 60th birthday and we thank him for a very interesting discussion on superselection rules.

\section{References}

[1] C.H. Townes and A.L. Schawlow, Microwave Spectroscopy (Mc Graw-Hill, New York, 1955)

[2] R.E. Weston, J. Am. Chem. Soc. 76, 2645 (1954).

[3] C. Constain and M. Sutherland, J. Phys. Chem. 56, 321 (1952).

[4] F. Hund, Z. Phys. series 43, 805 (1927).

[5] B. Bleaney and J.H. Loubster, Nature 161, 522 (1948).

[6] B. Bleaney and J.H. Loubster, Proc. Phys. Soc. (London) A63, 483 (1950).

[7] G. Birnbaum and A. Maryott, Phys. Rev. 92, 270 (1953).

[8] P.W. Anderson, Phys. Rev. 75, 1450 (1949).

[9] P. Pfeifer in Energy Storage and Redistribution in Molecules, Hinze Editor (Plenum, New York, 1983).

[10] A. Amann in Large Scale Molecular Systems: Quantum and Stochastic Aspects. Beyond the simple molecular picture, W. Gans, A. Blumen, and A. Amann Editors (Plenum Press, New York, 1991).

[11] A.S. Wightman, Some comments on the quantum theory of measurement in Probability methods in mathematical physics, F. Guerra, M. Loffredo, and C. Marchioro Editors (World Scientific, Singapore, 1992), p. 411.

[12] A.S. Wightman, Il nuovo Cimento 110 B, 751 (1995).

[13] P. Claverie and G. Jona-Lasinio, Phys. Rev. A 33, 2245 (1986).

[14] G. Jona-Lasinio and P. Claverie, Prog. Theor. Phys. Suppl. 86, 54 (1986).

[15] G. Jona-Lasinio, F. Martinelli, and E. Scoppola, Phys. Rep. 77, 313 (1981).

[16] G. Jona-Lasinio, F. Martinelli, and E. Scoppola, Commun. Math. Phys. 80, 223 (1981).

[17] G. Jona-Lasinio, C. Presilla, C. Toninelli, paper in preparation.

[18] P. de Smedt, P. Nielaba, J.L. Lebowitz, J. Talbot, and L. Dooms, Phys. Rev. A 38, 1381 (1988).

[19] J.P. Blaizot and G. Ripka, Quantum theory of finite systems (The MIT Press, Cambridge, Massachusetts, 1986).

[20] D.J. Caldwell and H. Eyring, The theory of optical activity (Wiley Interscience, New York, 1971).

[21] J. Kupsch, J. Math. Phys. 41, 5945 (2000). 
[22] W.H. Zureck, Phys. Rev. D 26, 1862 (1982).

[23] G. Lindblad, Comm. Math. Phys. 48, 119 (1976). 